\title{
CONVERGENCE OF STOCHASTIC PROCESSES
}

\author{
BY V. S. VARADARAJAN ${ }^{1}$
}

Communicated by J. L. Doob, December 30, 1960

1. Many problems in probability theory, when properly formulated, appear as problems in the theory of convergence of stochastic processes. The need for such a theory was demonstrated by the early results of Doob [4], Donsker [5] and others. In their fundamental papers, LeCam [10] and Prohorov [11] developed several aspects of such a theory. Their work was based on, and was a development of, the earlier work of A. D. Alexandrov [1] and Kolmogorov [9]. However, several questions which naturally arise were either not discussed or discussed only under unnecessary restrictions. The following remarks contain an outline of a general theory of measures on topological spaces. Only the statements and the appropriate formulations of the main results are given. The detailed proofs will be published elsewhere.

2. Let $X$ be a topological space and $C(X)$ the Banach space of bounded real-valued continuous functions on $X . S$ is the smallest $\sigma$-field of subsets of $X$ with respect to which all the elements of $C(X)$ are measurable. By measure we mean probability measures defined on $S$ and these arise, in the classical manner following F. Riesz, from linear functionals $\phi$ defined on $C(X)$. Given a nonnegative linear functional $\phi$ on $C(X)$ with $\phi(1)=1$, we have the representation

$$
\phi(f)=\int_{X} f d \mu
$$

for all $f \in C(X)$ with a (unique) measure $\mu$, provided $\phi$ is $\sigma$-smooth, i.e. for any sequence $\left\{f_{n}\right\}$ of elements of $C(X), \downarrow 0$ pointwise over $X, \phi\left(f_{n}\right) \rightarrow 0$. The set of all measures is denoted by $M(X)$, or simply by $M$, when there is no doubt as to what $X$ is.

$M$ is a subset of the dual-space of $C(X)$ and as such inherits the weak topology of the dual of $C(X)$. Our main concern is with the structure of this topology over $M$ and its subsets. The two main problems examined are the metrizability of $M$ and the structure of compact subsets of $M$.

1 This work was done during 1958-1959 while the author was in the Indian Statistical Institute, Calcutta, but due to diverse reasons the announcement was delayed up to now. 
3. We begin with a classification of measures. A measure $\mu$ is called $\tau$-smooth, if

$$
\int_{X} f_{j} d \mu \rightarrow 0
$$

for every net ${ }^{2}\left\{f_{j}\right\} \downarrow 0$ pointwise over $X$. The set of all $\tau$-smooth measures is denoted by $M_{\tau}$. A measure $\mu$ is called tight if

$$
\int_{X} \cdot d \mu
$$

is continuous on the unit sphere of $C(X)$ with respect to the topology of uniform convergence on compacta. The set of tight measures on $X$ is denoted by $M_{t}$. Clearly $M_{t}(X) \subset M_{\tau}(X) \subset M(X)$.

THEOREM 1. In order that a measure $\mu$ be $\tau$-smooth it is sufficient that there exists a closed Lindelöf ${ }^{2}$ subset $C$ of $X$ such that $\mu_{*}(X-C)=0$, $\mu_{*}$ denoting the inner measure induced by $\mu$. If $X$ is paracompact, this condition is necessary and sufficient.

In particular, if $X$ is a metric space, $\mu$ is $\tau$-smooth if and only if there exists a closed separable subset $C$ of $X$ such that $\mu(X-C)=0$.

It is interesting to examine the conditions under which we have the relation

$$
M=M_{\tau} .
$$

From Theorem 1 it follows at once that this is the case as soon as $X$ is a separable metric space.

THEOREM 2. If $X$ is a metric space, $M(X)=M_{\tau}(X)$ if and only if $M\left(X_{0}\right)=M_{\tau}\left(X_{0}\right)$ for every closed discrete subspace $X_{0}$ of $X$.

In other words $M(X)=M_{\tau}(X)$ if and only if the only measures defined on closed discrete subspaces of $X$ are those with mass concentrated on a countable set. It is well known $[2$, p. 187] that this question is intimately related with some questions in the theory of sets. In particular, it follows, on assuming the continuum hypothesis, that $M(X)=M_{\tau}(X)$ for any metric space of cardinality less than or equal to that of the continuum.

THEOREM 3. A measure $\mu$ is tight if and only if for each $\epsilon>0$ there exists a compact set $K_{\epsilon} \subset X$ such that

$$
\mu_{*}\left(X-K_{\epsilon}\right)<\epsilon .
$$

${ }^{2}$ See $[8]$ for this and other terminology concerning topological spaces. 
If $X$ is a complete metric space, $M_{t}=M_{\tau}$. In particular if $X$ is a separable and complete metric space, $M=M_{\tau}=M_{t}$.

4. The questions of metrizability and compactness in $M$ were first examined by $\mathrm{P}$. Levy in the case when $X$ is the real line (cf. for instance [6]) who proved that $M(X)$ in this case is a separable and complete $^{3}$ metric space. When $X$ is an arbitrary topological space, an examination of the imbedding

$$
\gamma: x \rightarrow \mu_{x},
$$

which sends $x \in X$ into the measure $\mu_{x}$ concentrated at $x$, reveals that for the metrizability of the space $M(X)$ one must have (i) $X$ is metrizable and (ii) $M(X)=M_{\tau}(X)$. In view of this and Theorem 2 it is thus natural to attempt to prove that $M_{\tau}$ is metrizable whenever $X$ is. We have

TheOREM 4. If $X$ is a metric space, $M_{\tau}(X)$ is metrizable. $M_{\tau}(X)$ is metrizable as a complete metric space when and only when $X$ is a complete metric space.

For the completeness part we note that the imbedding (3) of $X$ into $M_{\tau}(X)$ sends $X$ onto a closed subset of $M_{\tau}(X)$. On the other hand, if $X$ is complete, we introduce $\beta X$, its Stone-Cech compactification $(X \subset \beta X)$. By a general theorem due to Cech [3], $X$ is a $G_{\delta}$ in $\beta X$. Any measure $\mu$ on $X$ gives rise to a measure $\mu$ on $\beta X$ for which $\bar{\mu}(\beta X-X)=0$. It can be shown that $\mu$ is regular (in the sense of $[7$, p. 224]) if and only if $\mu \in M_{\tau}(X)$ and that the regular measures on $\beta X$ are precisely the $\tau$-smooth measures on $\beta X$. We thus obtain an imbedding of $M_{\tau}(X)$ into $M_{\tau}(\beta X)$. It can further be proved that this is a homeomorphism and that the image of $M_{\tau}(X)$ is a $G_{\delta}$ in $M_{\tau}(\beta X)$ (which is a compact Hausdorff space incidentally). Cech's theorem now assures us that $M_{\tau}(X)$ can be made complete under an equivalent metric.

We proceed next to a study of the compact subsets of $M$. In view of the nature of this communication we shall restrict ourselves to the case of greatest interest from the point of view of applications. A set $D \subset M(X)$ is called tight iff for each $\epsilon>0$ there exists a compact set $K . \subset X$ such that

$$
\operatorname{Sup}_{\mu \in D} \mu_{*}\left(X-K_{\epsilon}\right)<\epsilon,
$$

It is clear that only subsets of $M_{t}(X)$ can be tight sets; further,

${ }^{8}$ Completeness, here as elsewhere, is always completeness under some equivalent metric. 
it can be proved that a set $D \subset M_{t}$ is tight if and only if the corresponding set of linear functionals are equicontinuous at 0 in the topology (over the unit sphere of $C(X)$ ) of uniform convergence on compacta. The following theorem is easy to prove.

Theorem 5. If $D$ is tight, then $\bar{D} \subset M_{t}$ and is compact.

In [9] Kolmogorov raised the interesting question as to whether the converse of Theorem 5 is true. That this is so, when $X$ is a separable and complete metric space, was proved by Prohorov [11]. The following theorem settles the question when $X$ is an arbitrary metric space.

TheOREM 6. If $X$ is a metric space and $D$ is a compact subset of $M_{t}$, then $D$ is a tight set.

In view of well-known Ascoli theorems [8, p. 233] the proof proceeds by showing that the map

$$
(f, \mu) \rightarrow \int f d \mu
$$

of $C_{I}(X) X D$ into the reals $\left(C_{I}(X)\right.$ being the unit sphere of $X$ under the topology of uniform convergence on compacta) is continuous. We note now that $D$ is a metric space (Theorem 4 ) and hence convergence on $D$ is of a sequential nature. The desired continuity is now obtained by using a theorem of LeCam [10] (which is essentially Theorem 6 for the case when $D$ consists of a convergent sequence plus its limit point).

It might be remarked that when $X$ is not a metric space there are examples of compact subsets $D$ of $M_{t}$ which are not tight.

5. Applications to stochastic processes arise when we regard a stochastic process as a measure on a topological space $X$ of functions. If

$$
\xi, \xi_{1}, \xi_{2}, \cdots
$$

is a sequence of stochastic processes, the convergence of $\xi_{n}$, as $n \rightarrow \infty$, to $\xi$ then implies the convergence of the distribution of $g\left[\xi_{n}\right]$ to that of $g[\xi]$ for all continuous functions $g$ on $X$. Typical problems are those in which $X$ is a separable Banach space and $\xi$ is a random variable with values in $X$ which is normally distributed i.e. for any bounded linear functional $x^{*}$ on $X, x^{*}(\xi)$ is normally distributed. We then consider the central limit problem. Let

$$
\eta_{1}, \eta_{2}, \cdots
$$


be independent identically distributed $X$-valued random variables with $E\left(\eta_{i}\right) \equiv 0$. If

$$
\xi_{n}=\frac{1}{n}\left(\eta_{1}+\cdots+\eta_{n}\right),
$$

under what conditions do the distributions of $\xi_{n}$ converge in $X$ ? When $X$ is a Hilbert space, Prohorov proved [11] that $E\left\|\eta_{i}\right\|^{2}<\infty$ was a necessary and sufficient condition. The general problem when $X$ is an arbitrary separable Banach space remains unsolved, but the following theorem is one of several special results:

TheORem 7. If $X$ is the space $l_{1}$ (of all sequences

$$
\alpha=\left(a_{1}, a_{2}, \cdots\right)
$$

with $\sum_{n}\left|a_{n}\right|<\infty$ for which $\left.\|\alpha\|=\sum_{n}\left|a_{n}\right|\right)$, then

$$
\sum_{n}\left[\operatorname{Var} \eta_{1}^{(n)}\right]^{1 / 2}<\infty
$$

is a necessary and sufficient condition that $\xi_{n}$ should converge in distribution.

Here $\eta_{1}^{(n)}$ denotes the $n$th component of $\eta_{1}$ and Var denotes variance.

\section{REFERENCES}

1. A. D. Alexandrov, Additive set functions in abstract spaces, Mat. Sb. vol. 8 (1940) pp. 307-348; vol. 9 (1941) pp. 563-628; vol. 13 (1943) pp. 169-238.

2. G. Birkhoff, Lattice theory, Rev. ed., Amer. Math. Soc. Colloquium Publications, vol. 25, 1948.

3. E. Cech, On bicompact spaces, Ann. of Math. vol. 38 (1937) pp. 823-844.

4. J. L. Doob, Heuristic approach to the Kolmogorov-Smirnov theorems, Ann. Math. Statist. vol. 20 (1949) pp. 393-403.

5. M. Donsker, Justification and extension of Doob's heuristic approach to the Kolmogorov-Smirnov theorems, Ann. Math. Statist. vol. 23 (1952) pp. 277-281.

6. B. V. Gnedenko and A. N. Kolmogorov, Limit distributions for sums of independent random variables, Reading, Addison-Wesley, 1954.

7. P. R. Halmos, Measure theory, Princeton, Van Nostrand, 1950.

8. J. L. Kelley, General topology, Princeton, Van Nostrand, 1955.

9. A. N. Kolmogorov and Yu. V. Prohorov, Zufällige Funktionen und Grenzverteilungssätze, Bericht über die Tagung Wahrscheinlichkeitsrechnung und mathematische Statistik, Berlin, Deutscher Verlag der Wissenschaften, 1954, pp. 113-126.

10. L. LeCam, Convergence in distribution of stochastic processes, Univ. California Publ. Statist. vol. 2 (1957) pp. 207-236.

11. Yu. V. Prohorov, Convergence of random processes and limit theorems in probability theory, Teor. Veroyatnost. i Primenen. vol. 1 (1956) pp. 177-238.

The University of WashingtoN 